\title{
Incretin based drugs and the risk of pancreatic cancer: international multicentre cohort study
}

\author{
Laurent Azoulay, ${ }^{1,2}$ Kristian B Filion, ${ }^{1,3}$ Robert W Platt, ${ }^{4}$ Matthew Dahl, ${ }^{5}$ Colin R Dormuth, ${ }^{6}$ \\ Kristin K Clemens, ${ }^{7}$ Madeleine Durand, ${ }^{8}$ David N Juurlink, ${ }^{9}$ Laura E Targownik, 5,10 Tanvir C Turin, ${ }^{11}$ \\ J Michael Paterson, 9,12 Pierre Ernst' ${ }^{1,3}$ for the Canadian Network for Observational Drug Effect \\ Studies (CNODES) Investigators
}

For numbered affiliations see end of article

Correspondence to:

L Azoulay

laurent.azoulay@mcgill.ca

Additional material is published online only. To view please visit the journal online.

Cite this as: $B M / 2016 ; 352: i 581$ http://dx.doi.org/10.1136/bmj.i581 Accepted: 11 January 2016

\section{ABSTRACT}

\section{OBJECTIVE}

To determine whether the use of incretin based drugs compared with sulfonylureas is associated with an increased risk of incident pancreatic cancer in people with type 2 diabetes.

DESIGN

Population based cohort.

SETTING

Large, international, multicentre study combining the health records from six participating sites in Canada, the United States, and the United Kingdom.

\section{PARTICIPANTS}

A cohort of 972384 patients initiating antidiabetic drugs between 1 January 2007 and 30 June 2013, with follow-up until 30 June 2014.

\section{MAIN OUTCOME MEASURES}

Within each cohort we conducted nested case-control analyses, where incident cases of pancreatic cancer were matched with up to 20 controls on sex, age, cohort entry date, duration of treated diabetes, and duration of follow-up. Hazard ratios and $95 \%$ confidence intervals for incident pancreatic cancer were estimated, comparing use of incretin based drugs with use of sulfonylureas, with drug use lagged by one year for latency purposes. Secondary analyses assessed whether the risk varied by class (dipeptidyl peptidase- 4 inhibitors and glucagon-like peptide-1 receptor agonists) or by duration of use (cumulative duration of use and time since treatment initiation). Site specific hazard ratios were pooled using random effects models. RESULTS

During 2024441 person years of follow-up (median follow-up ranging from 1.3 to 2.8 years; maximum 8

\section{WHAT IS ALREADY KNOWN ON THIS TOPIC}

The safety of incretin based drugs is controversial, with some studies reporting an association with the incidence of pancreatic cancer

Additional observational studies are needed to assess whether these drugs are associated with this outcome

\section{WHAT THIS STUDY ADDS}

In this study, the use of incretin based drugs was not associated with an increased risk of pancreatic cancer, compared with sulfonylureas

The risk did not vary by duration of use or by drug type

Although the effects of incretin based drugs will require long term surveillance owing to the latency of cancer, these findings provide some reassurance on their potential association with pancreatic cancer

years), 1221 patients were newly diagnosed as having pancreatic cancer (incidence rate 0.60 per 1000 person years). Compared with sulfonylureas, incretin based drugs were not associated with an increased risk of pancreatic cancer (pooled adjusted hazard ratio $1.02,95 \%$ confidence interval 0.84 to 1.23 ). Similarly, the risk did not vary by class and evidence of a duration-response relation was lacking.

CONCLUSIONS

In this large, population based study the use of incretin based drugs was not associated with an increased risk of pancreatic cancer compared with sulfonylureas. Although this potential adverse drug reaction will need to be monitored long term owing to the latency of the cancer, these findings provide some reassurance on the safety of incretin based drugs.

\section{Introduction}

Incretin based drugs, which include dipeptidyl peptidase-4 (DPP-4) inhibitors and glucagon-like peptide-1 receptor agonists, are a relatively new group of drugs used to treat type 2 diabetes. These drugs have been associated with a lower risk of hypoglycaemia and with favourable effects on body weight ${ }^{1}$; however, concern has arisen that their use may be associated with the development of pancreatic cancer. ${ }^{23}$ This concern has been heightened by an analysis of the US Food and Drug Administration adverse events database, where the spontaneous reporting rate of pancreatic cancer was 2.9 and 2.7 times higher with exenatide and sitagliptin, respectively, compared with other oral antidiabetic drugs. ${ }^{4}$ In contrast, randomised controlled trials have not demonstrated this signal, ${ }^{5-11}$ although most of these trials were of short duration and none were designed or powered to assess the risk of pancreatic cancer.

To date, six observational studies have assessed the association between incretin based drugs and risk of pancreatic cancer. ${ }^{12-17}$ These studies have, however, provided conflicting results and several had methodological shortcomings. ${ }^{18}$ Given this controversy, regulatory agencies have called for additional studies of this potential association. ${ }^{19}$ To this end, the Canadian Network for Observational Drug Effect Studies (CNODES) ${ }^{20}$ designed a large, international, multicentre study to determine whether the use of incretin based drugs is associated with an increased risk of pancreatic cancer in people with type 2 diabetes. 


\section{Methods}

Data sources

This was a retrospective cohort study using a common analytical protocol to conduct studies using the administrative and electronic medical record databases from six CNODES participating sites across three countriesfour Canadian provinces (Alberta, Manitoba, Ontario, and Quebec), the United States (MarketScan), and the United Kingdom (Clinical Practice Research Datalink, CPRD). The Canadian databases include patient level information on physician billings, hospital admissions, and prescription drug claims. The Ontario data were restricted to patients aged 65 years or older. The US MarketScan database includes medical information for individuals and their dependents insured by private health insurance plans. The UK CPRD contains the complete primary care medical records for more than 13 million people enrolled in more than 680 general practices, and it has been shown to be representative of the UK population. ${ }^{21}$ The inclusion of the sites was based on an a priori estimation of their cohort size and the availability of incretin based drugs during the study period. Therefore, we did not include the provinces of Nova Scotia and Saskatchewan, two of the smaller CNODES sites. ${ }^{20}$ The British Columbia site was not included owing to lengthy timelines for access to the data.

\section{Study population}

Base cohort

In each participating site a base cohort was assembled consisting of all patients who commenced treatment with a first ever prescription for a non-insulin antidiabetic drug (metformin, sulfonylureas, thiazolidinediones, DPP-4 inhibitors, glucagon-like peptide-1 receptor agonists, $\alpha$ glucosidase inhibitors, and meglitinides) from the earliest date of data availability in each participating site up to 30 June 2013 (or the latest date of data availability in each participating site, whichever occurred first). The date of each patient's first prescription defined entry to the base cohort. We then sequentially excluded patients with the following characteristics at entry to the base cohort: age less than 18 years (or the minimum age for which prescription data are available plus 365 days), less than 365 days of medical history in the database before entry to the base cohort (to ensure no previous use of antidiabetic drugs and to assess comorbidities), previous insulin prescription (this may indicate more advanced disease), and women with a history of polycystic ovarian syndrome or a diagnosis of gestational diabetes in the 365 days before entry to the base cohort, as these conditions are other possible indications for metformin.

\section{Study cohort}

Within the base cohort we identified a study cohort consisting of all patients who initiated a new antidiabetic drug class any time after incretin based drugs entered the market in each participating site up until 30 June 2013 (or the latest date of data availability in each participating site). These new users included those who commenced treatment with an antidiabetic drug (that is, first ever prescription of a non-insulin antidiabetic drug), as well as those who added on or switched to an antidiabetic drug class not previously identified in their drug history. The date of this new prescription defined entry to the study cohort. We then sequentially excluded patients with a previous diagnosis of any cancer (other than non-melanoma skin cancer), those who had undergone pancreatectomy, and those with a history of congenital defects of the pancreas at any time before entry to the study cohort. Finally, we excluded patients with less than 365 days of follow-up after entry to the study cohort to ensure a minimum potential duration of drug use and to minimise protopathic bias from the inclusion of patients whose treatments might reflect type 2 diabetes secondary to pancreatic cancer. ${ }^{22}$

Thus, for all patients meeting the study inclusion criteria, the start of follow-up was set to 365 days after entry to the study cohort (that is, the start of person time at risk). They were followed until a diagnosis of incident pancreatic cancer, or censored on death from any cause, end of coverage in the database, or end of the study period (30 June 2014, or the latest date of data availability in each participating site), whichever occurred first.

\section{Case-control selection}

In each participating site we conducted nested case-control analyses within the study cohort. This approach was chosen because of the time varying nature of drug use, the size of the cohorts, and the relatively long duration of follow-up. ${ }^{23}$ We used risk set sampling for the matching of controls to cases, a method that produces odds ratios that are unbiased estimators of hazard ratios. ${ }^{23-25}$

Cases consisted of all patients with a hospital admission (lasting at least one day) for pancreatic cancer during follow-up (international classification of diseases, ninth revision codes 157.0-157.9; and 10th revision code C25.x, in either the primary or secondary position). This outcome definition has excellent positive and negative predictive values (88.2\% and 99.9\%, respectively; unpublished data) compared with the Ontario cancer registry. ${ }^{26}$ For each case we defined the index date by the date of hospital admission.

We randomly selected up to 20 controls for each case, matched on age (365 days either way), sex, date of entry to the study cohort (180 days either way), duration of treated diabetes before entry to the study cohort (defined as time between entry to the base cohort and entry to the study cohort; 90 days either way), and duration of follow-up. Matched controls were assigned the index date of their respective cases.

\section{Drug groups}

Cases and matched controls were classified into one of three mutually exclusive drug groups, defined hierarchically by use of incretin based drugs (DPP-4 inhibitors (linagliptin, sitagliptin, vildagliptin, and saxagliptin) or glucagon-like peptide-1 receptor agonists (exenatide, liraglutide)), alone or in combination with other antidiabetic drugs; sulfonylureas, alone or in combination with other antidiabetic drugs; and other antidiabetic 
drugs. We defined the use of these antidiabetic drug classes as receiving at least one prescription at any time before the year immediately preceding the index date (that is, prescriptions received during a one year lag period were ignored for latency purposes and to minimise reverse causality).

The reference category for all analyses was use of sulfonylureas, a drug class typically used in the second line to third line setting, and thus at a similar stage in the clinical course of diabetes as incretin based drugs. ${ }^{27}$ In contrast, comparing incretin based drugs with metformin (a first line treatment) or insulin (a last line treatment) may introduce time lag bias, a form of confounding by indication that may be difficult to adjust for in statistical models. ${ }^{28}$ Finally, thiazolidinediones have fallen out of favour because of possible adverse events (such as cardiovascu$\operatorname{lar}^{29}$ and cancer risks ${ }^{30}$ ), and thus do not represent the most clinically relevant comparator group.

\section{Statistical analysis}

Based on the Poisson distribution, we calculated the crude incidence rate of pancreatic cancer with 95\% confidence intervals. We used conditional logistic regression to estimate hazard ratios and corresponding 95\% confidence intervals of incident pancreatic cancer, comparing use of incretin based drugs with use of sulfonylureas. This was considered the prespecified primary analysis.

All models were adjusted for potential confounders measured at entry to the study cohort, including alcohol related disorders, history of acute or chronic pancreatitis, and use of statins at any time before entry to the study cohort. We also adjusted for the presence of microvascular complications of diabetes (neuropathy, renal disease, retinopathy, and peripheral arteriopathy; measured at any time before entry to the study cohort) and the number of unique antidiabetic drugs received in the 365 days before entry to the study cohort (entered

Patients with first ever prescription for non-insulin antidiabetic drug ( $n=6580331)$

Excluded ( $\mathrm{n}=4530245)$ :

$<18$ years or minimum age $(n=280336)$

$<365$ days coverage in database $(n=4016126)$

Date inconsistencies $(n=22672)$

Insulin before first ever non-insulin antidiabetic drug ( $\mathrm{n}=124012)$

Women with diagnosed polycystic ovarian syndrome $(n=67517)$

Women with gestational diabetes in year before first prescription $(n=19582)$

Patients included in base cohort $(n=2050086)$

Excluded $(n=400284)$

Died or left cohort before first incretin based drug entered market $(n=155696)$

Never added on or switched to new antidiabetic drug class after incretin based drugs entered market $(n=244588)$

Cohort of new users or switchers after incretin based drugs entered market $(n=1649802)$

Excluded $(n=677418)$ :

Diagnosis of pancreatic cancer, pancreatectomy, or diagnosis of congenital

defects of pancreas before study cohort entry $(n=154$ 596)

Patients with $\ll 365$ days of follow-up after study cohort entry $(n=522822)$

Patients included in study cohort $(n=972384)$

Fig 1 | Flow of base and study cohorts as a four level ordered categorical variable), both as proxies for diabetes severity. In addition, we adjusted the models for two general measures of comorbidity (total number of hospital admissions and total number of unique non-diabetic drugs prescribed, both measured in the 365 days before entry to the study cohort; both entered as four level ordered categorical variables). ${ }^{31}$ In the CPRD, we additionally adjusted the models for body mass index $\left(<25 \mathrm{~kg} / \mathrm{m}^{2}, 25-29 \mathrm{~kg} / \mathrm{m}^{2}, \geq 30 \mathrm{~kg} /\right.$ $\mathrm{m}^{2}$; last measure before entry to the study cohort), haemoglobin A1c level ( $\leq 7 \%$ (53 mmol/mol), 7.1-8.0\% (54-64 $\mathrm{mmol} / \mathrm{mol}),>8 \%$ (64 mmol $/ \mathrm{mol})$; last measure before entry to the study cohort), and smoking status (ever, never). Given that information was missing in relatively few patients (between $0.4 \%$ and $13.8 \%$ ) we modelled these variables including a category for missing values.

\section{Secondary analyses}

We conducted four prespecified secondary analyses. In the first, we stratified incretin based drugs as DPP-4 inhibitors (linagliptin, saxagliptin, sitagliptin, vildagliptin) and glucagon-like peptide-1 receptor agonists (exenatide, liraglutide). In the remaining three analyses we tested for a duration-response relation between use of incretin based drugs and pancreatic cancer. In the first of these analyses, we assessed whether the risk of pancreatic cancer varied with cumulative duration of use. For this analysis we calculated cumulative duration as the total duration of all prescriptions received from entry to the study cohort to the index date (classified as $<1$ year, 1-1.9 years, and $\geq 2$ years). Similarly, we modelled the time since initiation of treatment, defined as the time between the first ever prescription of an incretin based drug and the index date (classified as 1-1.9 years and $\geq 2$ years). Finally, we repeated the cumulative duration of use and time since initiation analyses for DPP-4 inhibitors and glucagon-like peptide-1 receptor agonists, separately.

\section{Sensitivity analyses}

We conducted four sensitivity analyses to assess the robustness of our findings. Firstly, to account for uncertainty in the length of the possible latency period and to assess the impact of protopathic bias, we varied the lag period before the index date to six months and two years. Secondly, we replicated the primary analysis using a stricter exposure definition, requiring at least four prescriptions within any 12 month period between entry to the study cohort and the year immediately before the index date. Thirdly, we repeated the primary analysis comparing use of incretin based drugs with use of sulfonylureas among those who initiated sulfonylureas on or after entry to the study cohort, since some sulfonylurea users may have initiated treatment before entry to the study cohort. Finally, we repeated the primary analysis with a reduced set of covariates to facilitate model convergence at all participating sites.

We used DerSimonian and Laird random effects models $^{32}$ with inverse variance weighting to pool site specific estimates. The estimates were also pooled using fixed 


\begin{tabular}{|c|c|c|}
\hline Baseline characteristics & Cases & Controls \\
\hline No of patients & 1221 & 22298 \\
\hline \multicolumn{3}{|l|}{ CNODES site: } \\
\hline US MarketScan & $568(46.5)$ & $11349(50.9)$ \\
\hline Quebec & $251(20.6)$ & $4135(18.5)$ \\
\hline UK Clinical Practice Research Datalink & 209 (17.1) & $3473(15.6)$ \\
\hline Ontario & $95(7.8)$ & $1725(7.7)$ \\
\hline Alberta & $59(4.8)$ & 1180 (5.3) \\
\hline Manitoba & $39(3.2)$ & $436(2.0)$ \\
\hline Mean age (years): & 68.2 & 68.1 \\
\hline $18-25$ & $S^{*}$ & $9(0.0)$ \\
\hline $26-35$ & $\mathrm{~S}^{\star}$ & $31(0.1)$ \\
\hline $36-45$ & $18(1.5)$ & $344(1.5)$ \\
\hline 46-55 & $147(12)$ & $2904(12.5)$ \\
\hline $56-65$ & $315(25.8)$ & $5730(25.5)$ \\
\hline $66-75$ & $418(34.2)$ & $7769(34.6)$ \\
\hline$\geq 76$ & $320(26.2)$ & $5511(25.8)$ \\
\hline Men & $671(55.0)$ & $12379(55.0)$ \\
\hline \multicolumn{3}{|l|}{ Year of study cohort entry: } \\
\hline 2007 & $204(16.7)$ & $3827(16.5)$ \\
\hline 2008 & $200(16.4)$ & $3613(16.9)$ \\
\hline 2009 & $280(22.9)$ & $5134(22.8)$ \\
\hline 2010 & $260(21.3)$ & $4703(21.0)$ \\
\hline 2011 & $194(15.9)$ & $3784(17.0)$ \\
\hline 2012 & $\mathrm{~S}^{*}$ & $1227(5.9)$ \\
\hline 2013 & $\mathrm{~S}^{*}$ & $12(0.1)$ \\
\hline Mean duration of treated diabetes (years) & 1.5 & 1.5 \\
\hline \multicolumn{3}{|l|}{ Body mass index $\left(\mathrm{kg} / \mathrm{m}^{2}\right)$ : } \\
\hline$<25$ & $32(15.3)$ & $394(12.0)$ \\
\hline $25-29$ & $73(34.9)$ & $1186(35.5)$ \\
\hline$\geq 30$ & $96(45.9)$ & $1830(50.9)$ \\
\hline Missing & $8(3.8)$ & $63(1.5)$ \\
\hline \multicolumn{3}{|l|}{ Haemoglobin A1c: } \\
\hline$\leq 7 \%(53 \mathrm{mmol} / \mathrm{mol})$ & $24(11.5)$ & $504(14.4)$ \\
\hline $7.1-8.0 \%(54-64 \mathrm{mmol} / \mathrm{mol})$ & $54(25.8)$ & $1044(29.3)$ \\
\hline$>8 \%(64 \mathrm{mmol} / \mathrm{mol})$ & $104(49.8)$ & $1353(42.5)$ \\
\hline Missing & $27(12.9)$ & $572(13.8)$ \\
\hline Alcohol related disorders & $37(3.0)$ & $541(2.6)$ \\
\hline \multicolumn{3}{|l|}{ Smoking status: } \\
\hline Ever & $134(64.1)$ & $2108(40.1)$ \\
\hline Never & $\mathrm{S}^{\star}$ & $1350(59.5)$ \\
\hline Missing & $\mathrm{S}^{\star}$ & $15(0.4)$ \\
\hline Statins & $764(62.6)$ & $13761(63.6)$ \\
\hline Acute or chronic pancreatitis & $43(3.5)$ & $224(1.2)$ \\
\hline Neuropathy & $36(2.9)$ & $488(2.9)$ \\
\hline Renal disease & $120(9.8)$ & $1885(10.1)$ \\
\hline Retinopathy & $140(11.5)$ & $2486(12.6)$ \\
\hline Peripheral arteriopathy & $87(7.1)$ & $1310(6.0)$ \\
\hline Mean No of hospital admissions & 0.2 & 0.2 \\
\hline \multicolumn{3}{|l|}{ No of hospital admissions: } \\
\hline 0 & $1023(83.8)$ & $19365(86.2)$ \\
\hline 1 & $151(12.4)$ & $2317(10.7)$ \\
\hline 2 & $33(2.7)$ & $463(2.2)$ \\
\hline 3 & $12(1.0)$ & $94(0.5)$ \\
\hline$\geq 4$ & $7(0.6)$ & $58(0.4)$ \\
\hline Mean No of unique non-antidiabetic drugs & 8.8 & 8.3 \\
\hline \multicolumn{3}{|l|}{ No of unique non-antidiabetic drugs: } \\
\hline 0 & $72(5.9)$ & $1802(7.4)$ \\
\hline 1 & $52(4.3)$ & $979(4.1)$ \\
\hline 2 & $61(5.0)$ & $1177(5.1)$ \\
\hline 3 & $61(5.0)$ & $1374(5.9)$ \\
\hline$\geq 4$ & $976(79.9)$ & $19966(77.4)$ \\
\hline
\end{tabular}

effects modelling in sensitivity analyses. Heterogeneity between sites was estimated using the $\mathrm{I}^{2}$ statistic.

\section{Patient involvement}

Our study was a secondary data analysis and did not include patients as study participants. No patients were involved in setting the research question or the outcome measures, nor were they involved in the design and implementation of the study. There are no plans to involve patients in the dissemination of results, nor will we disseminate results directly to patients, beyond our general media communications plan.

\section{Results}

The study cohort included 972384 patients initiating antidiabetic drugs (fig 1). The mean age at entry to the study cohort was 56.9 years, $50.9 \%$ were men, and the median duration of follow-up across participating sites ranged from 1.3 to 2.8 years, not including the one year follow-up required for the purposes of latency (see supplementary table 1 for additional baseline characteristics of the study cohort). Overall, the study cohort generated 2024441 person years of follow-up. During this time 1221 patients were newly diagnosed as having pancreatic cancer (cases), generating a crude incidence rate of 0.60 (95\% confidence interval 0.57 to 0.64 ) per 1000 person years (see supplementary table 2 for site specific incidence rates).

Table 1 shows the baseline characteristics of the cases and 22298 matched controls. Compared with controls, cases were less likely to be obese but were more likely to have uncontrolled diabetes, alcohol related disorders, ever smoked, and a history of acute or chronic pancreatitis. The cases and matched controls were similar on the other characteristics.

Overall, compared with users of sulfonylureas users of incretin based drugs were younger, had longer durations of treated diabetes, were more likely to be obese, and had higher haemoglobin A1c levels. In contrast, users of incretin based drugs were less likely to have been diagnosed as having microvascular complications of diabetes. Both groups had similar histories of pancreatitis (see supplementary table 3).

Table 2 presents the results of the primary and secondary analyses. Compared with use of sulfonylureas, use of incretin based drugs was not associated with an increased risk of incident pancreatic cancer (pooled adjusted hazard ratio 1.02, 95\% confidence interval 0.84 to 1.23; fig 2). Similarly, there was no association in the secondary analysis, which categorised the use of incretin based drugs by class (DPP-4 inhibitors, 1.02, 0.84 to 1.24; glucagon-like peptide-1 receptor agonists, $1.13,0.38$ to 3.38 ). In other secondary analyses, a cumulative duration of less than one year yielded an increased but non-significant pooled hazard ratio (1.53, 0.93 to 2.51), whereas longer durations of use generated pooled hazard ratios close to or below unity (1-1.9 years, $1.07,0.82$ to 1.39 ; $\geq 2$ years, $0.62,0.36$ to 1.07$)$. The analysis of time since initiation yielded pooled hazard ratios close to the null (1-1.9 years, 1.06, 0.86 to 1.31 ; $\geq 2$ years, $0.93,0.60$ to 1.45 ). 


\begin{tabular}{|c|c|c|}
\hline Baseline characteristics & Cases & Controls \\
\hline Mean No of prestudy cohort entry antidiabetic drugs & 0.3 & 0.3 \\
\hline \multicolumn{3}{|l|}{ Prestudy cohort entry antidiabetic drugs: } \\
\hline 0 & $994(81.4)$ & $19829(81.5)$ \\
\hline 1 & $117(9.6)$ & $1470(10.7)$ \\
\hline 2 & $66(5.4)$ & $615(4.5)$ \\
\hline 3 & $34(2.8)$ & $252(2.2)$ \\
\hline$\geq 4$ & $8(0.7)$ & $132(1.1)$ \\
\hline \multicolumn{3}{|l|}{ Study cohort entry drugs } \\
\hline Metformin & $853(69.9)$ & $16824(70.5)$ \\
\hline Sulfonylureas & $278(22.8)$ & 4024 (20.0) \\
\hline Thiazolidinediones & $72(5.9)$ & $1235(6.0)$ \\
\hline DPP-4 inhibitors & $85(7.0)$ & $1521(7.3)$ \\
\hline GLP-1 receptor agonists & $6(0.5)$ & $125(1.4)$ \\
\hline$\alpha$ glucosidase inhibitors & $\mathrm{S}^{*}$ & $59(0.4)$ \\
\hline Meglitinides & $17(1.4)$ & $188(1.2)$ \\
\hline Insulins & $43(3.5)$ & $223(2.1)$ \\
\hline Others & $\mathrm{S}^{*}$ & $43(0.2)$ \\
\hline \multicolumn{3}{|c|}{$\begin{array}{l}\text { CNODES=Canadian Network for Observational Drug Effect Studies; DPP-4=dipeptidyl peptidase-4; GLP-1=glucagon- } \\
\text { like peptide-1. } \\
\text { *When the total across participating sites was <6, data were suppressed (denoted by S) owing to privacy } \\
\text { restrictions. When summing data across sites, we assigned a value of } 3 \text { to small cells ( } \leq 5) \text {. As such, the sum of } \\
\text { count data may differ from the presented total. } \\
\text { tMeans and proportions among controls were weighted by number of controls per case and then weighted by } \\
\text { number of cases per site. } \\
\text { ¥Matching variable. } \\
\text { \$Data from UK Clinical Practice Research Datalink; percentages based on } 209 \text { cases and } 3473 \text { matched controls } \\
\text { 9Non-mutually exclusive categories. }\end{array}$} \\
\hline
\end{tabular}

Table 2 Association between use of incretin based drugs and incidence of pancreatic cancer. Values are numbers (percentages) unless stated otherwise

\begin{tabular}{|c|c|c|c|c|c|}
\hline Exposuret & $\begin{array}{l}\text { Cases } \\
(n=1221)\end{array}$ & $\begin{array}{l}\text { Controls } \\
(n=22298)\end{array}$ & $\begin{array}{l}\text { Crude } \\
\text { hazard ratio }\end{array}$ & $\begin{array}{l}\text { Adjusted hazard } \\
\text { ratio }(95 \% \mathrm{CI})\end{array}$ & $I^{2}(\%)$ \\
\hline \multicolumn{6}{|l|}{ Primary analysis: } \\
\hline Sulfonylureas & $439(36.0)$ & $5851(26.2)$ & 1.00 & 1.00 (Reference) & \multirow[t]{2}{*}{0.0} \\
\hline Incretin based drugs & $200(16.4)$ & $2800(12.6)$ & 1.01 & $1.02(0.84$ to 1.23$)$ & \\
\hline \multicolumn{6}{|l|}{ Class of incretin based drug: } \\
\hline DPP-4 inhibitors & $183(15.0)$ & $2543(11.4)$ & 1.02 & 1.02 (0.84 to 1.24$)$ & 0.0 \\
\hline GLP-1 receptor agonists & $17(1.4)$ & $257(1.2)$ & 1.11 & 1.13 (0.38 to 3.38$)$ & 72.0 \\
\hline \multicolumn{6}{|c|}{ Cumulative duration of use (years): } \\
\hline$<1$ & $80(6.6)$ & $1012(4.5)$ & 1.52 & $1.53(0.93$ to 2.51$)$ & 61.5 \\
\hline $1-1.9$ & $86(7.0)$ & $1183(5.3)$ & 1.05 & $1.07(0.82$ to 1.39$)$ & 0.0 \\
\hline$\geq 2$ & $34(2.8)$ & $605(2.7)$ & 0.64 & $0.62(0.36$ to 1.07$)$ & 34.9 \\
\hline \multicolumn{6}{|l|}{ Time since initiation (years): } \\
\hline $1-1.9$ & $149(12.2)$ & $2126(9.5)$ & 1.05 & 1.06 (0.86 to 1.31$)$ & 0.0 \\
\hline$\geq 2$ & $51(4.2)$ & $674(3.0)$ & 0.96 & $0.93(0.60$ to 1.45$)$ & 27.3 \\
\hline
\end{tabular}

DPP-4=dipeptidyl peptidase-4; GLP-1=glucagon-like peptide-1.

${ }^{*}$ Cases and controls matched on sex, age, year of entry to study cohort, duration of treated diabetes, and duration of follow-up.

tTable displays 639 cases and 8651 controls exposed to sulfonylureas and incretin based drugs. Users of other antidiabetic drugs (corresponding to 582 cases and 13647 controls) are not displayed, but were considered in the regression model for proper estimation of treatment effects.

$\ddagger$ Adjusted for alcohol related disorders, history of acute or chronic pancreatitis, microvascular complications of diabetes (neuropathy, renal disease, retinopathy, and peripheral arteriopathy), number of hospital admissions $(0,1,2,3, \geq 4)$, number of unique non-diabetic drugs in previous year $(0,1,2,3, \geq 4)$, number of antidiabetic drugs received before entry to study cohort $(0,1,2,3, \geq 4)$, ever use of statins. In the Clinical Practice Research Datalink, models were further adjusted for body mass index, smoking status, and glycated haemoglobin A1c level ( $\leq 7.0 \%$ (53 mmol $/ \mathrm{mol}), 7.1-8.0 \%(54-64 \mathrm{mmol} / \mathrm{mol}),>8.0 \%(64 \mathrm{mmol} / \mathrm{mol})$ ).

$\S$ Analysis limited to UK Clinical Practice Research Datalink and the US MarketScan, two sites where GLP-1

receptor agonists were available during the study period

Similar findings were observed with each class of incretin based drug (see supplementary figures 1-17).

Sensitivity analyses

Figure 3 shows the results of the sensitivity analyses. Overall, these analyses yielded findings that were

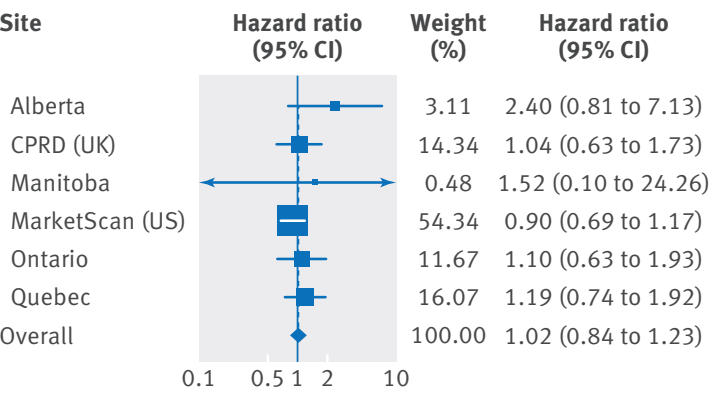

Fig 2 | Forest plot of association between use of incretin based drugs and risk of pancreatic cancer among patients with type 2 diabetes. Reference category was use of sulfonylureas. Box size is proportional to weight of participating site in random effects meta-analysis $\left(\mathrm{I}^{2}=0 \%\right)$

consistent with those of the primary analysis (see supplementary figures 18-23). The pooled adjusted hazard ratio in the fixed effects model was identical to that generated with the random effects model for the primary analysis (1.02, 0.80 to 1.23$)$.

\section{Discussion}

In this large population based study we investigated the association between the use of incretin based drugs and the risk of pancreatic cancer. The findings of this study, comprising a combined cohort of almost one million people with type 2 diabetes, suggest that use of incretin based drugs is not associated with an increased risk of pancreatic cancer compared with use of sulfonylureas. These results remained consistent in several secondary and sensitivity analyses, which considered type of incretin based drugs and duration of use.

\section{Comparison with other studies}

To our knowledge six observational studies have assessed the association between the use of incretin based drugs and the incidence of pancreatic cancer. ${ }^{12-17}$ Overall, most of these studies did not report statistically significant associations. ${ }^{12-15} 17$ One study reported an overall increased risk with sitagliptin (hazard ratio 1.40, 95\% confidence interval 1.13 to 1.75), but with no clear dose-response relation. ${ }^{16}$ However, these studies had some methodological shortcomings, including the potential inclusion of prevalent (rather than new) users, ${ }^{13} 14$ protopathic bias, ${ }^{1617}$ lack of an appropriate comparator group, ${ }^{13} 1617$ small sample sizes, ${ }^{12-15}$ and limited durations of follow-up. ${ }^{12-16}$ Moreover, three studies were limited to specific incretin based drugs: exenatide, ${ }^{12} 13$ liraglutide, ${ }^{14}$ and sitagliptin. ${ }^{16}$

Our null findings are consistent with those of recent large randomised controlled trials of DPP-4 inhibitors and glucagon-like peptide-1 receptor agonists: saxagliptin, alogliptin, sitagliptin, and lixisenatide. ${ }^{8-11}$ However, these randomised controlled trials generated a total of only 51 pancreatic cancer events and were thus not statistically powered to detect a moderate increased risk of this rare outcome. ${ }^{8-11}$ Specifically, in the Saxagliptin Assessment of Vascular Outcomes Recorded in Patients with Diabetes Mellitus-Thrombolysis in Myocardial Infarction 
Sensitivity analysis

6 month lag period

2 year lag period

Stricter exposure definition

Sulfonylurea initiation after study cohort entry

Reduced model $1^{*}$

Reduced model $2 \dagger$

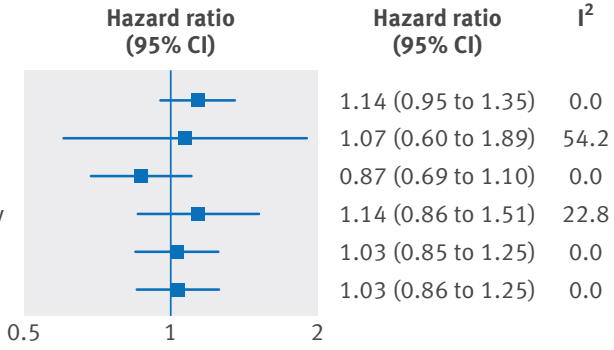

Fig 3 | Sensitivity analyses for association between use of incretin based drugs and incidence of pancreatic cancer. Reference category for all analyses was use of sulfonylureas. Site specific hazard ratios were pooled using random effects meta-analysis. Stricter exposure definition: defined as receiving at least four prescriptions within any 12 month period. *Adjusted for alcohol related disorders, history of acute or chronic pancreatitis, composite variable of microvascular complications of diabetes, number of hospital admissions (continuous), number of unique non-diabetic drugs in previous year (continuous), number of antidiabetic drugs received before entry to study cohort (continuous), and ever use of statins. In CPRD, we further adjusted for body mass index, smoking status, and glycated haemoglobin A1c level ( $\leq 7.0 \%$ (53 mmol $/ \mathrm{mol}), 7.1-8.0 \%$ (54-64 $\mathrm{mmol} / \mathrm{mol})$, and $>8.0 \%$ (64 mmol $/ \mathrm{mol})$ ). †Adjusted for Deyo version of Charlson comorbidity index and history of acute or chronic pancreatitis

(SAVOR-TIMI) 53 trial, 16 pancreatic cancer events occurred (five in the saxagliptin group versus 11 in the placebo group) after a median follow-up of 2.1 years. ${ }^{8}$ In the Examination of Cardiovascular Outcomes with Alogliptin versus Standard of Care (EXAMINE) trial, no pancreatic cancer events occurred in either the alogliptin group or the placebo group after a median follow-up of 18 months. ${ }^{9}$ In the Trial Evaluating Cardiovascular Outcomes with Sitagliptin (TECOS) trial, 23 pancreatic cancer events occurred (nine in the sitagliptin group versus 14 in the placebo group) after a median follow-up of 3.0 years. ${ }^{10}$ Finally, in the Evaluation of Lixisenatide in Acute Coronary Syndrome (ELIXA) trial, 12 pancreatic cancer events occurred (three in the lixisenatide group versus nine in the placebo group) after a median follow-up of 25 months. ${ }^{11}$

\section{Strengths and limitations of this study}

Our study was specifically designed to circumvent the methodological limitations of the aforementioned observational studies ${ }^{12-15}$ and randomised controlled trials. ${ }^{8-11}$ Firstly, the use of population based cohorts from six participating sites across three countries strengthens the generalisability of our findings. Secondly, with an overall sample size of close to one million patients followed for up to eight years, our primary analysis was well powered to detect modest but clinically important associations. Thirdly, all patients entering the base and study cohorts were new users of antidiabetic drugs, thus avoiding biases related to the inclusion of prevalent users. ${ }^{33}$ Fourthly, confounding by indication was minimised through matching, statistical adjustment, and by comparing users of incretin based drugs with users of sulfonylureas, a drug class sharing similar indications. ${ }^{27}$ Finally, latency and protopathic bias were addressed by excluding all exposures initiated in the year before the index date; findings were generally consistent in sensitivity analyses in which the duration of the lag period was varied.
Our study has some limitations. Firstly, although we found that our case definition based on a hospital admission for pancreatic cancer had high positive and negative predictive values $(88.2 \%$ and $99.9 \%$, respectively), misclassification of the outcome is possible. However, it is unlikely that this misclassification would be differential between users of incretin based drugs and users of sulfonylureas. Furthermore, our overall incidence rate of pancreatic cancer $(0.60,95 \%$ confidence interval 0.57 to 0.64 per 1000 person years) is consistent with the incidence rate reported in a Canadian study that linked a cohort of patients with type 2 diabetes to the British Columbia Cancer Agency database (0.65, 0.59 to 0.71 per 1000 person years). ${ }^{34}$ Secondly, given the observational nature of the study, residual confounding by disease severity remains possible. We addressed this by matching cases and controls on duration of treated diabetes, a proxy for disease severity, ${ }^{35}$ as well as adjusting the models for the presence of microvascular complications of type 2 diabetes and number of antidiabetic drugs received before entry to the study cohort. It was not possible to adjust for certain pancreatic cancer risk factors such as physical activity, occupational exposures, diet, and family history. However, as achieving glycaemic control is the main motivation for prescribing incretin based drugs or sulfonylureas, we do not believe these unmeasured variables are differentially distributed across the exposure groups. Thirdly, in the secondary analysis of cumulative duration of use, we observed a high hazard ratio for less than one year (pooled adjusted hazard ratio $1.53,95 \%$ confidence interval 0.93 to 2.51 ). This association is of questionable plausibility given the limited duration of exposure and may point to some residual protopathic bias. Specifically, it is possible that some patients were prescribed incretin based drugs because of worsening glycaemic control as a result of early signs of pancreatic cancer, but were switched to step-up treatment (such as insulin) after failing to achieve glycaemic control in the first months of use. It is also important to note that although the primary analysis was well powered, secondary analyses such as those of duration of use were based on fewer events, thus generating wider confidence intervals. Finally, incretin based drugs are relatively new treatments, limiting the potential duration of follow-up. Importantly, the previous signal was based on spontaneous reports that occurred relatively soon after the availability of incretin based drugs. ${ }^{4}$ Thus, our null findings are important and argue against a tumour promoter effect. None the less, given the latency of pancreatic cancer, this association will need to be reassessed once these drugs have been on the market for a longer period.

\section{Conclusions and implications}

In this large, population based study, we found that the use of incretin based drugs is not associated with an overall increased risk of pancreatic cancer, compared with the use of sulfonylureas in patients followed for up to eight years (median 1.3-2.8 years). These findings remained consistent by class and by duration of use. 
Although the upper limit of the confidence interval was sufficient to exclude a $24 \%$ increased risk of pancreatic cancer, there is a need for the continued surveillance of this adverse drug effect, particularly given the latency of the cancer. None the less, this study should provide some reassurance to patients and clinicians with concerns of an association between these drugs and pancreatic cancer.

\section{AUTHOR AFFILIATIONS}

${ }^{1}$ Center for Clinical Epidemiology, Lady Davis Research Institute, lewish General Hospital, Montreal, Quebec, H3T 1E2, Canada ${ }^{2}$ Department of Oncology, McGill University, Montreal, Canada ${ }^{3}$ Department of Medicine, McGill University, Montreal, Canada 4Departments of Pediatrics and of Epidemiology, Biostatistics, and Occupational Health, McGill University, and the Research Institute of the McGill University Health Centre, Montreal, Canada

${ }^{5}$ Manitoba Centre for Health Policy, Department of Community Health Sciences, University of Manitoba, Winnipeg, Canada and Department of Family Medicine, McMaster University, Hamilton, Canada

${ }^{6}$ Department of Anesthesiology, Pharmacology \& Therapeutics, University of British Columbia, Vancouver, Canada

${ }^{7}$ Department of Medicine, Western University, London, Canada ${ }^{8}$ Department of Internal Medicine, Centre Hospitalier de l'Université de Montréal, Montreal, Canada

9Institute for Clinical Evaluative Sciences, Toronto, Canada

${ }^{10}$ Section of Gastroenterology, Division of Internal Medicine, University of Manitoba, Winnipeg, Canada

${ }^{11}$ Department of Family Medicine, University of Calgary, Calgary, Canada

${ }^{12}$ Institute of Health Policy, Management and Evaluation, University of Toronto, Toronto

We are grateful for the programming support of Zhihai Ma and lianguo Zhang(Alberta), Gregory A Carney (US MarketScan), Hui Yin (UK CPRD), Caixia Fangyun Wu and Simon Hollands (Ontario), and Menglan Pang (Quebec). We also thank Corine Mizrahi, Melissa Dahan, and Laura Sang at the Coordinating Center; Héloïse Cardinal for her contribution to the development of the study design; and the important contributions of the CNODES collaborators and assistants at each site. This study was made possible through data sharing agreements between CNODES member research centres and the respective provincial governments of Alberta, Manitoba (HIPC No 2014/2015-08; HREB No H2014:236), Ontario, and Quebec. The opinions, results, and conclusions reported in this paper are those of the authors. No endorsement by the provinces is intended or should be inferred. KBF holds a Canadian Institutes of Health Research new investigator award. RWP is supported by a Chercheur-National Award of the Fonds de Recherche du Quebec-Santé (FRQS; Quebec Foundation for Health Research). MD is supported by a clinical investigator award of the FRQS. DNJ is supported by the Eaton scholar programme, Department of Medicine, University of Toronto. The Canadian Network for Observational Drug Effect Studies (CNODES) Investigators are: Samy Suissa (principal investigator); Colin R Dormuth (British Columbia); Brenda R Hemmelgarn (Alberta); Gary F Teare (Saskatchewan); Patricia Caetano, and Dan Chateau (Manitoba); David A Henry and J Michael Paterson (Ontario); Jacques LeLorier (Québec); Adrian R Levy (Nova Scotia); Pierre Ernst (UK Clinical Practice Research Datalink (CPRD)); Robert W Platt (Methods); and Ingrid S Sketris (Knowledge Translation). CNODES, a collaborating centre of the Drug Safety and Effectiveness Network (DSEN), is funded by the Canadian Institutes of Health Research (grant No DSE-111845).

Contributors: All authors conceived and designed the study, analysed and interpreted the data, and critically revised the manuscript for important intellectual content. LA, KBF, CRD, MD, LT, TCT, JMP, and PE acquired the data. LA drafted the manuscript. LA, KBF, RWP, MD, CRD, $M D, L E T, T C T, J M P$, and PE carried out the statistical analysis. The Canadian Network for Observational Drug Effect Studies Investigators obtained the funding. LA, KBF, RWP, and PE supervised the study. LA is the guarantor.

Funding: Canadian Network for Observational Drug Effect Studies, a collaborating centre of the Drug Safety and Effectiveness Network, is funded by the Canadian Institutes of Health Research (grant No DSE-111845). The sponsor had no influence on the design and conduct of the study; collection, management, analysis, and interpretation of the data; and preparation, review, or approval of the manuscript. This study was made possible through data sharing agreements between CNODES member research centres and the respective provincial governments of Alberta, Manitoba, Ontario, and Quebec. The opinions, results, and conclusions reported in this paper are those of the authors. No endorsement by the provinces is intended or should be inferred.

Competing interests: All authors have completed the ICMJE uniform disclosure form at www.icmje.org/coi_disclosure.pdf (available on request from the corresponding author) and declare: LET is on the speaker's panel for Janssen Canada, Takeda Canada, Pfizer Canada, and Shire Canada, has received grant support from Pfizer Canada and Abbvie Canada, and is on advisory boards for Takeda Canada, Abbvie Canada, and Janssen Canada. RWP received consulting fees for work unrelated to this project from Pfizer, Amgen, Abbvie, and Novartis.

Ethical approval: The study protocol was approved by the institutional review boards at all participating sites, and by the Independent Scientific Advisory Committee (ISAC) of the CPRD (protocol number 14 119R). The ISAC protocol was made available to the journal reviewers. The study protocol is registered at www. clinicaltrials.gov (NCT02475499).

Data sharing: No additional data available.

Transparency: The guarantor (LA) affirms that this manuscript is an honest, accurate, and transparent account of the study being reported; that no important aspects of the study have been omitted; and that any discrepancies from the study as planned (and, if relevant, registered) have been explained.

This is an Open Access article distributed in accordance with the Creative Commons Attribution Non Commercial (CC BY-NC 3.0) license, which permits others to distribute, remix, adapt, build upon this work non-commercially, and license their derivative works on different terms, provided the original work is properly cited and the use is non-commercial. See:http://creativecommons.org/licenses/ by-nc/3.0/.

1 Holst J). The physiology of glucagon-like peptide 1. Physiol Rev 2007;87:1409-39. doi:10.1152/physrev.00034.2006.

2 Gale EA. GLP-1-based therapies and the exocrine pancreas: more light, or just more heat?Diabetes 2012;61:986-8. doi:10.2337/ db11-1838.

3 Kabuzek K, Kozłowski M, Szkudłapski D, Sikorska P, Kozłowska M Okopien B. Incretin-based therapies in the treatment of type 2 diabetes-more than meets the eve?Eur / Intern Med 2013:24:20712. doi:10.1016/j.ejim.2013.01.009.

4 Elashoff M, Matveyenko AV, Gier B, Elashoff R, Butler PC. Pancreatitis, pancreatic, and thyroid cancer with glucagon-like peptide-1-based therapies. Gastroenterology 2011;141:150-6. doi:10.1053/j. gastro.2011.02.018. 21334333.

5 Shyangdan DS, Royle PL, Clar C, Sharma P, Waugh NR. Glucagon-like peptide analogues for type 2 diabetes mellitus: systematic review and meta-analysis. BMC Endocr Disord 2010;10:20. doi:10.1186/1472-6823-10-20.

6 Monami M, Dicembrini I, Martelli D, Mannucci E. Safety of dipeptidyl peptidase-4 inhibitors: a meta-analysis of randomized clinical trials. Curr Med Res Opin 2011;27(Suppl 3):57-64. doi:10.1185/03007995.2 011.602964

7 Jeong KH, Yoo BK. The efficacy and safety of liraglutide. Int/ Clin Pharm 2011;33:740-9. doi:10.1007/s11096-011-9552-8.

8 Scirica BM, Bhatt DL, Braunwald E, et al. SAVOR-TIMI 53 Steering Committee and Investigators. Saxagliptin and cardiovascular outcomes in patients with type 2 diabetes mellitus. N EnglJ Med 2013;369:1317-26. doi:10.1056/NEJMoa1307684.

9 White WB, Cannon CP, Heller SR, et al. EXAMINE Investigators. Alogliptin after acute coronary syndrome in patients with type 2 diabetes. N EnglJ Med 2013;369:1327-35. doi:10.1056/ NEJMoa1305889.

10 Green IB, Bethel MA, Armstrong PW, et al. TECOS Study Group. Effect of sitagliptin on cardiovascular outcomes in type 2 diabetes. N Engl/ Med 2015;373:232-42. doi:10.1056/NEJMoa1501352.

11 Pfeffer MA, Claggett B, Diaz R, et al. ELIXA Investigators. Lixisenatide in patients with type 2 diabetes and acute coronary syndrome. N Engl J Med 2015;373:2247-57. doi:10.1056/NEJMoa1509225.

12 Dore DD, Seeger JD, Chan KA. Incidence of health insurance claims for thyroid neoplasm and pancreatic malignancy in association with exenatide: signal refinement using active safety surveillance. Ther $A d v$ Drug Saf 2012;3:157-64. doi:10.1177/2042098612446473.

13 Romley A, Goldman DP. Solomon M, McFadden D, Peters AL. Exenatide therapy and the risk of pancreatitis and pancreatic cancer in a privately insured population. Diabetes Technol Ther 2012;14:904-11. doi:10.1089/dia.2012.0075. 
14 Funch D, Gydesen H, Tornøe K, Major-Pedersen A, Chan KA. A prospective, claims-based assessment of the risk of pancreatitis and pancreatic cancer with liraglutide compared to other antidiabetic drugs. Diabetes Obes Metab 2014;16:273-5. doi:10.1111/dom.12230.

15 Gokhale M, Buse JB, Gray CL, Pate V, Marquis MA, Stürmer T. Dipeptidyl-peptidase-4 inhibitors and pancreatic cancer: a cohort study. Diabetes Obes Metab 2014;16:1247-56. doi:10.1111/ dom.12379.

16 Tseng $\mathrm{CH}$. Sitagliptin and pancreatic cancer risk in patients with type 2 diabetes. Eur/Clin Invest 2016:46:70-9 doi:10.1111/eci.12570.

17 Knapen LM, van Dalem J, Keulemans YC, et al. Use of incretin agents and risk of pancreatic cancer: a population-based cohort study. Diabetes Obes Metab 2015; published online 5 November. doi:10.1111/dom.12605.

18 Azoulay L. Incretin-based drugs and adverse pancreatic events: almost a decade later and uncertainty remains. Diabetes Care 2015:38:951-3. doi:10.2337/dc15-0347.

19 Egan AG, Blind E, Dunder K, et al. Pancreatic safety of incretin-based drugs-FDA and EMA assessment. N Engl J Med 2014;370:794-7. doi:10.1056/NEIMp1314078.

20 Suissa S, Henry D, Caetano P, et al. Canadian Network for Observational Drug Effect Studies (CNODES). CNODES: the Canadian Network for Observational Drug Effect Studies. Open Med 2012;6:e134-40.

21 Herrett E, Gallagher AM, Bhaskaran K, et al. Data resource profile: Clinical Practice Research Datalink (CPRD). Int J Epidemiol 2015:44:827-36. doi:10.1093/ije/dyv098.

22 Pannala R, Basu A, Petersen GM, Chari ST. New-onset diabetes: potential clue to the early diagnosis of pancreatic cancer. Lancet Oncol 2009;10:88-95. doi:10.1016/S1470-2045(08)70337-1.

23 Suissa S. Novel approaches to pharmacoepidemiology study design and statistical analysis. In: Strom B, ed. Pharmacoepidemiology.4th ed. Wiley, 2005: 811-29.

24 Essebag V, Platt RW, Abrahamowicz M, Pilote L. Comparison of nested case-control and survival analysis methodologies for analysis of time-dependent exposure. BMC Med Res Methodol 2005;5:5. doi:10.1186/1471-2288-5-5.

25 Suissa S. The quasi-cohort approach in pharmacoepidemiology: upgrading the nested case-control. Epidemiology 2015;26:242-6 doi:10.1097/EDE.0000000000000221.
26 Hall S, Schulze K, Groome P, Mackillop W, Holowaty E. Using cancer registry data for survival studies: the example of the Ontario Cance Registry. JClin Epidemiol 2006;59:67-76. doi:10.1016/j. jclinepi.2005.05.001

27 Inzucchi SE, Bergenstal RM, Buse JB, et al. American Diabetes Association (ADA) European Association for the Study of Diabetes (EASD). Management of hyperglycemia in type 2 diabetes: a patient-centered approach: position statement of the American Diabetes Association (ADA) and the European Association for the Study of Diabetes (EASD). Diabetes Care 2012:35:1364-79. doi: $10.2337 /$ dc12-0413.

28 Suissa S, Azoulay L. Metformin and the risk of cancer: time-related biases in observational studies. Diabetes Care 2012;35:2665-73. doi:10.2337/dc12-0788.

29 Nissen SE, Wolski K. Effect of rosiglitazone on the risk of myocardial infarction and death from cardiovascular causes. N Engl) Med 2007:356:2457-71. doi:10.1056/NEIMoa072761.

30 Azoulay L, Yin H, Filion KB, et al. The use of pioglitazone and the risk of bladder cancer in people with type 2 diabetes: nested case-control study. BMJ 2012;344:e3645. doi:10.1136/bmj.e3645.

31 Schneeweiss S, Seeger JD, Maclure M, Wang PS, Avorn J, Glynn RJ. Performance of comorbidity scores to control for confounding in epidemiologic studies using claims data. Am Epidemiol 2001:154:854-64. doi:10.1093/aje/154.9854.11682368.

32 DerSimonian R, Laird N. Meta-analysis in clinical trials. Control Clin Trials 1986;7:177-88. doi:10.1016/0197-2456(86)90046-2.

33 Ray WA. Evaluating medication effects outside of clinical trials: new-user designs. Am J Epidemiol 2003;158:915-20. doi:10.1093/ aje/kwg231.

34 Johnson JA, Bowker SL, Richardson K, Marra CA. Time-varying incidence of cancer after the onset of type 2 diabetes: evidence of potential detection bias. Diabetologia 2011;54:2263-71. doi:10.1007/s00125-011-2242-1.

35 Benoit SR, Fleming R, Philis-Tsimikas A, Ji M. Predictors of glycemic control among patients with type 2 diabetes: a longitudinal study. BMC Public Health 2005;5:36. doi:10.1186/1471-2458-5-36.

Web extra: supplementary information 